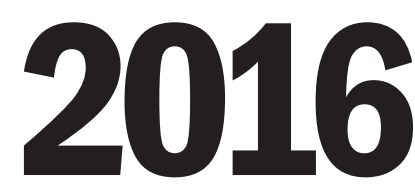

volume 13 | issue 1

an open access journal for architectural research

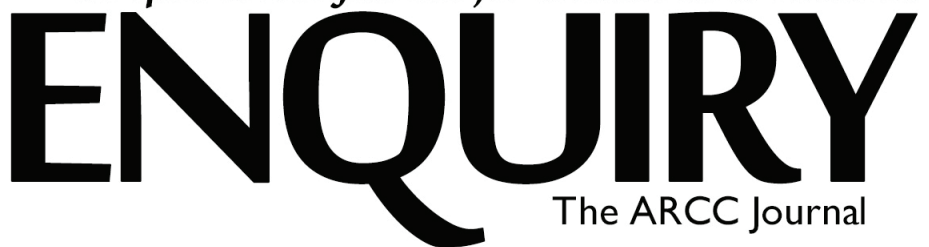

\title{
Research in Architectural Education: Theory and Practice of Visual Training
}

\author{
Kristin Jones
}

\begin{abstract}
Today, the significance of vision is often considered from multiple points of view including perceptual, cognitive, imaginative, historical, technical, ethical, cultural, and critical perspectives. Visual Studies, Visual Communication and Visual Design are popular courses of study found in many programs of higher education. This paper centers on a course called Visual Training within the domain of architectural education.

To illustrate the pedagogical significance of the 78-year old practice, a methodology of Visual Training as it has been conducted at Illinois Institute of Technology is presented. The paper describes the program of exercises used, and through an interpretation of the course outcomes, it reveals the course structure and pedagogical theory. The discussion shows how Visual Training establishes grounds for architectural critique based on visual perception and aesthetic judgment. In looking at this case of Visual Training, the paper revisits some of the fundamental premises of architectural pedagogy - from methods to ideals - and challenges assumptions about the role of vision in education by calling attention to existing biases shaping many of today's programs.
\end{abstract}

Keywords: visual training, architectural education, pedagogical research

\section{Permissions and copyright}

Authors retain copyright and grant the journal right of first publication with the work simultaneously licensed under a Creative Commons Attribution License that allows others to share the work with an acknowledgement of the work's authorship and initial publication in this journal.

Attribution-NonCommercial-ShareAlike 4.0 International (CC BY-NC-SA 4.0)

You are free to: Share - copy and redistribute the material in any medium or format. Adapt — remix, transform, and build upon the material for any purpose. You may not use the material for commercial purposes. The licensor cannot revoke these freedoms as long as you follow the license terms.

Under the following terms: Attribution - You must give appropriate credit, provide a link to the license and indicate if changes were made. You may do so in any reasonable manner, but not in any way that suggests the licensor endorses you or your use.

No additional restrictions - You may not apply legal terms or technological measures that legally restrict others from doing anything the license permits.

\section{How to cite:}

Jones, Kristin. 2016. "Research in Architectural Education: Theory and Practice of Visual Training." Enquiry 13 (1): 7-16. http://dx.doi.org/10.17831/enq:arcc.v13i2.404

\section{INTRODUCTION}

Today, the significance of vision is often considered from multiple points of view including perceptual, cognitive, imaginative, historical, technical, ethical, cultural, and critical perspectives. Visual Studies, Visual Communication and Visual Design are popular courses of study found in many programs of higher education including schools of architecture. "Visual studies" is a broad term for courses in which issues of visual ontology and visual culture are analyzed and/or critiqued. The following excerpt from University of Pennsylvania's Department of Visual Studies outlines the field of study from outside the domain of architecture:

New technologies and philosophies of vision influence how we see ourselves and our world, and how we think about seeing itself. The Visual Studies Major was created in 2003 to allow students to directly engage these developments through a multi-disciplinary course of study, connecting the theory, practice, and culture of seeing. (University of Pennsylvania 2016)

Within architecture programs, the terms "visual communication" and "visual design" are most often used for courses involving the production of visual culture. Visual Communication courses often focus on visual means of presenting and conveying nonverbal and verbal information. The teaching of drawing conventions (e.g. plans, elevations, sections, isometric, perspective, etc.) often characterizes this category of courses. The focus of Visual Design courses tends to be on creative visual expression. Mixed media, including physical and digital technologies, are often included.

This paper brings to light a lesser known course of study in architectural education called Visual Training, which aims to sharpen visual perception and enhance aesthetic judgment. A program for Visual Training was founded at Illinois Institute of Technology (IIT) in 1938-39 by Walter Peterhans within the curriculum of Mies van der Rohe. Since then, the teaching of Visual Training has continued at IIT. Very little has been written on Visual Training and the course name appears to be unique in the field.

Research was undertaken to understand and appreciate Visual Training from a pedagogical perspective. Bearing in mind the living history of 

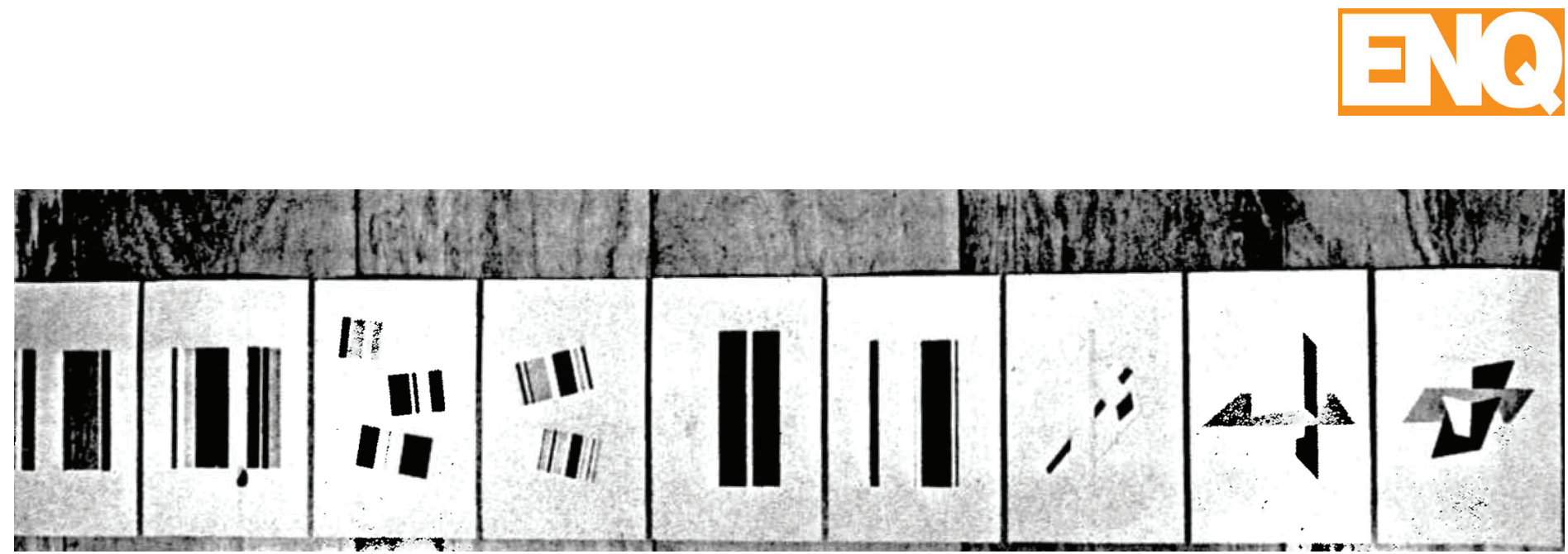

Figure 1. Visual Training exhibit, IIT ca. 1944 (Dearstyne 1944).

the course, this paper presents a methodology of Visual Training which includes descriptions of the existing program of exercises, their conduct, interpretations of course outcomes, and the course logic or structure. The educational theory underlying the 78-year old practice becomes more evident within this methodology. The paper concludes with a discussion about the role of vision in education which challenges existing biases shaping many of today's programs of architectural education.

\section{PRACTICE OF VISUAL TRAINING Exercises}

Visual Training was introduced to IIT in 1938-39 by Walter Peterhans (1897-1960). ${ }^{1}$ At first, it was implemented through drawing and photographic exercises. It is most likely that the drawing exercises were related to descriptive geometry (which Peterhans also taught) and that the photographic exercises were drawn from Peterhans' Bauhaus photography courses. But unfortunately, no examples of exercises from this period were found to corroborate this guess. In any case, within the first few years, the drawing and photographic exercises were replaced by exercises in a variety of media.

Over a period of 10 years or so, the focus of the course description shifted away from technical procedures like drawing and photography and toward visual sensibilities. By 1949, the general thrust of the course and the program of exercises were more or less established. ${ }^{2}$ Since then, the course description has remained unchanged.

Aesthetic expression as experience. Exercises in the study of form: proportion and rhythm, texture and color, mass and space. Exercises in visual perception and aesthetic judgment. Isolation and analysis; interdependence and integration of sensuous qualities. Aesthetic unity under restrictive conditions.

- Visual Training course description since 1949

\footnotetext{
${ }^{1}$ Mies van der Rohe invited Peterhans and Ludwig Hilberseimer to help found the architecture school at IIT. Peterhans was a commercial and artistic photographer educated in Germany in math, art history and philosophy, and was the Director of the Photography Department and founder of the Photography Workshop at the Bauhaus from 1929-1933.

${ }^{2}$ According to memory reports of former students and the IIT course bulletins.
}

In the following 10 years, the lessons matured and by the time Peterhans passed away in 1960, the program had been developed sufficiently enough to be carried on by his students.

Ten basic exercises for Visual Training were developed. Each problem has distinct visual objectives, specific elements of emphasis, varied procedural requirements and limitations, and technical problems (Peterhans 1958). Each exercise is conducted on $20^{\prime \prime} \times 30^{\prime \prime}$ white illustration board with one exception, a three-dimensional exercise. The boards are all the same size, color and finish, and are viewed in the vertical orientation, to control the number of variables having to be considered.

The first six problems examine the primary ways we define space in two dimensions. Three exercises deal with different means of subdividing finite (Euclidean) space and three deal with defining infinite (nonEuclidean) space. The first three exercises look at space divided into a) upper-lower and left-right (orthogonality), b) bottom-middle-top (interlaced rhythms), and c) major-minor (order). The next three look at space developed with d) one line continuously varying in direction and width (continuity + variation), e) multiple planes (discreteness + relativity), and f) the hyperbolic paraboloid (discreteness + continuity).

These problems are limited to black on white to focus on the graphical aspect, except for the study of planes, which introduces color as a factor. These exercises use paper cut with a sharp knife as a medium, except for the study of hyperbolic paraboloids, which is drawn in pencil. They focus on developing a sense of space and precise linear or graphical expression. They emphasize consistency and straightness in straight lines, fluidity and smoothness in curved lines, and clarity and nuance in distinctions.

The next two problems examine direct and indirect means of influencing liquid form. One exercise involves a wet-on-dry technique in the study of ink applied with an implement. The problem is limited to black ink. The other exercise involves a wet-on-wet technique, in which water applied to the board surface is used to spread pigment by hydrodynamic action. This problem is open to color and different types of liquid media including inks, watercolors, and acrylics may be used. Both exercises study fluid behavior, the interaction of liquid media with the white surface of the board and the overt and hidden influences on this interaction. 
The last two problems are characterized by the use of solid, textured materials. One is a two-dimensional problem focusing on appreciating the visual qualities of a variety of materials of different colors, textures and finishes by coordinating a set of twelve thin pieces. The other is a three-dimensional problem looking at composing of a variety of materials in different colors and textures arranged on a base. Whereas the "magic" of the two-dimensional exercises often happens in the transformation of the board surface into pictorial space (i.e. depth, movement), in the three-dimensional exercise, one has to overcome the concreteness of the materials in order to transform the space into something special, and deliver a sense of being in the presence of something clearly defined, yet not of material character. The threedimensional problem provides a transition from the two-dimensional board exercises to architectural space problems.

\section{Conduct}

The distinctive conduct of the Visual Training exercises was developed on account of both conceptual and cultural aims. On one hand, the exercises should enable students to understand the potential for expression by developing an understanding of the boundaries and dynamics of a problem. On the other, they are intended to develop a sense of quality including aesthetic judgment. In order to achieve both aims, the Visual Training exercises have historically been introduced in such a way that the student has no choice but to confront and consider the whole realm of possibilities and exert aesthetic judgment, followed by critique.

San Utsunomiya, IIT Associate Professor Emeritus and Visual Training instructor from 1966-1994, explained that the initial instructions he gave were to "describe the white area of the board with [for example] a line that continuously changes direction and thickness" (Utsunomiya 1977). Such an open-ended question is used in conjunction with dialogue and critique in order to develop an understanding of formal possibilities while, at the same time, embedding a sense of personal accountability for aesthetic judgment. Beginning with the students' solutions to this open question allows a pure initial assessment, because it demonstrates the current status of both technical ability and aesthetic judgment. The exercises are then directed at addressing specific areas for development.

The aim of the exercises is not to develop a design for something or to make the solutions look "like" anything else but aesthetic expression. ${ }^{3}$ But aesthetic expression comes in many shapes and sizes, and is difficult to provide criteria for without pinning down the outcomes. The sense of beauty upon which Visual Training was originally founded was described by Peterhans in terms of architecture in the following excerpt:

In a particular building firmness and commodity, structural soundness and fitness for service, may have been achieved. But soundness of structure, by itself, does not provide yet convenience. Equally, firmness and commodity do not grant yet delight. The structure must be so arranged as to obtain firmness; it must be

\footnotetext{
${ }^{3}$ Aesthetic expression is a term used to denote expressions of beauty, as well as other qualities that stir the human spirit, such as the sublime.
}

so ordered as to provide expected services; it must take such shape as to instill delight. One and the same elements articulate the structure, render its functions intelligible, and unite in a configuration of moving eloquence. Beauty, in particular, is not an article that can be obtained separately and affixed to a building; it can, however, be extracted and founded upon the elements that comprise the structure. (Peterhans 1955)

The nature of beauty, or delight (i.e. Venustas), ${ }^{4}$ so described is of an essential and integral kind. It is not like an ornament which can be "applied", but rather, is drawn out of the bounds of the problem itself. Hence, one of the primary aims of Visual Training is to begin to see and understand this integral aspect of beauty.

Visual Training does not approach this understanding through reading or criticizing others' past or current works, although corollary readings, videos and discussions are sometimes helpful. Instead, the program offers a break-down of various aspects of visual form and formal analysis in limited exercises. The conduct of these exercises involves a few distinctive features - primary among them is a process of refinement.

The exercises sharpen visual acuity by looking at qualities such as similarity and difference, consistency and straightness, clarity and fluidity. This sensitivity is then called on in the development of aesthetic judgment. As the proportional distribution of these qualities is analyzed and critiqued, an understanding of formal concepts such as balance, movement, rhythm and space are developed and enriched.

Visual comparison is a method which has been used in varying degrees by different instructors to develop understanding. It is a useful tactic for sensitizing the eye to difference and for opening the imagination to a realm of possibilities. The use of visual comparison in the analysis of art traces back to the early 20th century when Heinrich Wölfflin, Roger Fry, and others sought to point out formal differences between works of different artists and times. ${ }^{5}$ Two distinct types of visual comparison are found in the Visual Training pedagogy. One type is used to "open the eyes" or to develop a sense of a realm of possibilities, and the other to distinguish nuance.

Dialogical comparison is a specific type of visual comparison which serves an eye-opening function. A dialogical comparison is a comparison of two possible extremes. Once two extremes have been defined, a whole range of possibilities becomes comprehensible or visualizable by logical inference. Figure 2 shows two uses of the dialogical comparison. ${ }^{6}$ One example is seen in the upper right plate, where two extremes are arbitrated in one form. Another example is seen in the upper right pair of plates. The two plates illustrate a comparison of the distribution of weight on two halves of an S-shaped curve. The left plate includes more similar weight distribution throughout, and the right plate distributes

\footnotetext{
${ }^{4}$ See Vitruvius' Ten Books on Architecture (Vitruvius and Morgan 2005).

${ }^{5}$ See Principles of Art History: The problem of the development of style (Wölfflin and Hottinger 1950) and Vision and Design (Fry 2011).

${ }^{6}$ Dialogical comparisons, like those illustrated in Figure 2, are found consistently among the lessons of Peterhans.
} 
weight very differently on the two sides of the curve. Between two plates, a wide range of possibilities is implied. Likewise, the upper left pair of plates compares more similar (left) and more contrasting (right) distribution of rectangular proportions, more similar (left) versus more contrasting (right) line weight, and vertical (left) versus horizontal (right) emphasis. The two outer plates in the lower row contrast a preponderance of black with a preponderance of white. The two center plates in the lower row illustrate alternates in hierarchical order to these two extremes. Through visual dialog, these examples show the range of possibilities in the first four exercises.

Side-by-side comparison is another method used to compare and qualify difference. In Visual Training, side-by-side comparison can be used to develop the ability to see what each formal element is contributing to the whole and to appreciate the interdependence of all the elements comprising a composition. The method can be used in progressively finer-grained analyses. Figure 3 demonstrates the use of this method to clarify understanding of proportion with respect to the subdivided areas in the first Visual Training exercise.

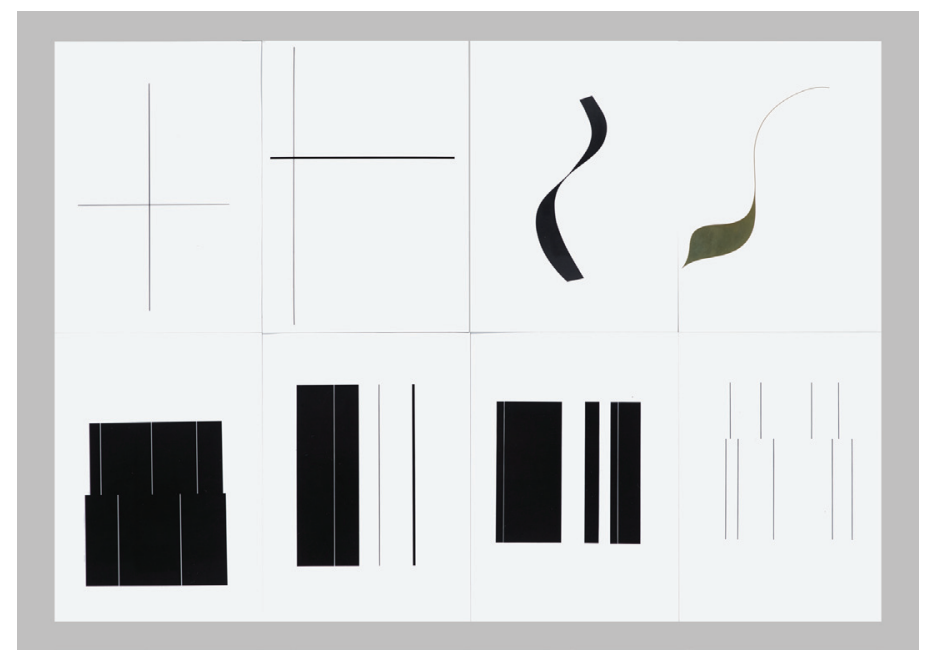

Figure 2. Photographs of final Visual Training plates, ca. 1950s. (Image courtesy of Walter Peterhans Estate, Museum Folkwang Essen.)

Say, for example, a student begins with the solution shown in Figure 3b. The subdivision of the compositional space in the left-right direction is clearly distinct. The ratio of upper to lower space is also decidedly different. However, the width of the spaces at the left and bottom edges of the plate, while different, are not highly differentiated from each other. This closeness causes a perceptual grouping emphasizing the left-bottom relation, which detracts from the overall aesthetic unity. The instructor may question these proportions and ask the student to clarify them by studying more contrast. The left plate (Fig. 3a) is a comparison with the vertical line shifted left, and the right plate (Fig. 3c) is a comparison with the horizontal shifted up. Both of these alternates increase the amount of contrast in proportion at the edges and overall. The alternates are therefore both clearer in comparison to the original which had more ambiguous differences in proportion. The result is a more balanced composition where less emphasis on the left-bottom relation allows greater expression of the unity of the whole.

These visual comparisons can be used at every step leading up to a final solution. For this exercise, the student would next look at line weight and contrast in line weight, using the same method to deal with ambiguity and uncertainty. Side-by-side comparisons demonstrate what each element contributes to the whole and supply the necessary visual evidence for grounding aesthetic judgment.

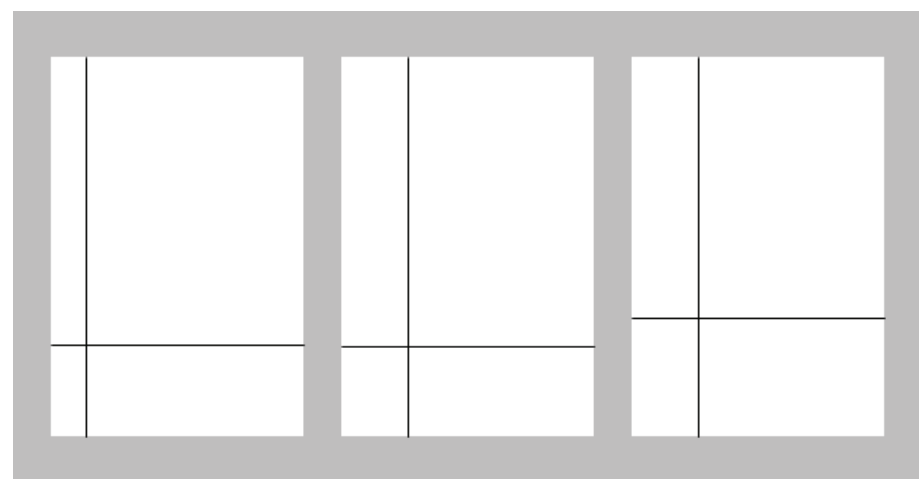

Figures 3. Diagrams $(a, b, c)$ illustrating comparative analysis.

Using these tactics, the exercises train visual perception to alternate between seeing the absolute values to seeing the aesthetic manifold, or the compositional whole. In order to develop aesthetic judgment, they shift focus from looking at the particulars in isolation and evaluating them as absolutes (e.g. I like this color), to looking at them diametrically and evaluating them with respect to a realm of possibilities (e.g. I like this color, but it is too weak here). This sense of possibilities in a realm establishes the conceptual framework to attach perceptual status, or a sense of expression, to absolute values (e.g. 1/4" is too strong, I should try $\left.3 / 16^{\prime \prime}\right)$. As students exert aesthetic judgment, analyze the results and receive critique, they develop intuition.

The process of refining aesthetic expression, as a reflection of aesthetic judgment, has much to do with mastering formal dynamics. For many of us, the process of refinement has to do with removing what is undesirable or questionable from the composition, as these prevent inherent beauty from shining through. As Francis Ford Coppola simply put:

I am a cinema lover, a wine lover and a coffee lover. Whether film, wine or coffee, the creative journey is really quite similar. You must start with gathering good raw material: grapes, coffee beans, or the scenes and fragments of the film. Then, from among them, you keep only the great and discard the not-so-good. Using skill and intuition, you must maximize what you have. It's a special kind of alchemy. (Coppola 2016)

The Visual Training exercises were developed in much the same spirit. Not only were the problems designed to provide access to what ties the 
arts together, their common foundations (Peterhans 1953), the exercises were designed to cultivate just this kind of intuition.

When an instructor identifies an area needing attention, or further development, a specific element which could have a positive impact on the outcome is targeted for further study. To observe the impact of a change, a student produces one or more alternate plates for comparison with the original. In the alternates, the overall visual effect of changing an isolated element is analyzed and the less desirable result is discarded. Visual comparison and analysis used in this way develops the ability to see what each element is contributing to the whole and to appreciate the interdependence of all the elements comprising a composition.

These methods of comparison are used along with questioning and critique to develop an appreciation for the dynamics of nonverbal expression, and to discover through the essential qualities of form, materials and technique, that sense of infinite possibility latent within the boundaries of a problem.

\section{INTERPRETING OUTCOMES OF VISUAL TRAINING}

Two generations of IIT alumni have continued Peterhans' Visual Training program - his students and their students. Although the same exercises have been used, the solutions found in the IIT collection of student work are not all the same. ${ }^{7}$ The final solutions kept in the IIT archive are usually simple, asymmetrical solutions executed with an expert technical standard. Among these exemplary boards, most of the formal differences can be explained by pedagogy.

One of the main reasons for variations in outcomes is that students are developing their own judgment, and to do so, the work must be their own. Hence, all the work is original and unique. The focus of Visual Training is the enhancement of visual perception and aesthetic judgment, not the production of superb boards for public consumption, as noted in Mies' description of Visual Training:

Although specially gifted students sometimes produced plates that would have enriched the collection of a museum, the purpose of the course was never to produce works of art, but to train the eyes. (Mies van der Rohe 1965)

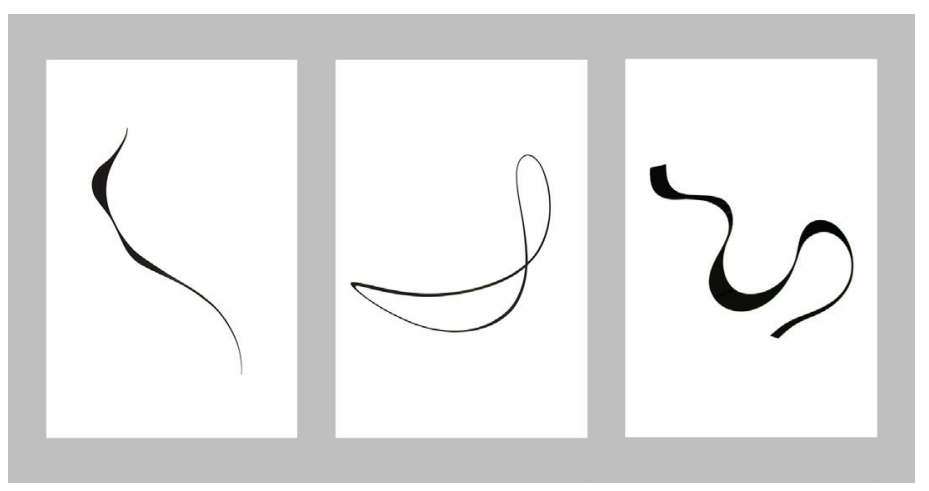

Figure 5. Examples. (Images courtesy of IIT College of Architecture. Photos by Tom Harris, Hedrich Blessing Photographers.)

This non-absolute and student-centered aim puts onus on the instructor to raise individual levels of ability and sensitivity to higher levels of achievement and understanding, not just to improve the look of a specific board. Because the path of each exercise is tailored to improving students' abilities, differentiated results are to be expected.

That being said, one technique to help students who are struggling is to limit the problem. For example, in a problem dealing with a question of color, a student might begin the exercise by limiting the study to black and white or one family of hues. Examples of such variations are found within the IIT collection of plates. The theoretical justification for permitting this variation is that the aesthetic quality can be more fully developed within a narrower scope. It is preferable that a student not become accustomed to submitting unresolved or insufficient work because the scope is overwhelming.

As mentioned earlier, some of the final plates can be interpreted as demonstrating an understanding of the boundaries of the problem (as in Figure 2). Other examples found in the collection, however, do not showcase a logical dichotomy or a comparison of two extremes. Some examples express a more free or irregular quality (as in Figure 5). In these examples, distinctions between similarity and contrast, opposition and mediation, are not expressed for their own sakes - as formal dialectics. In these examples, the formal variation results in a less "logical" and
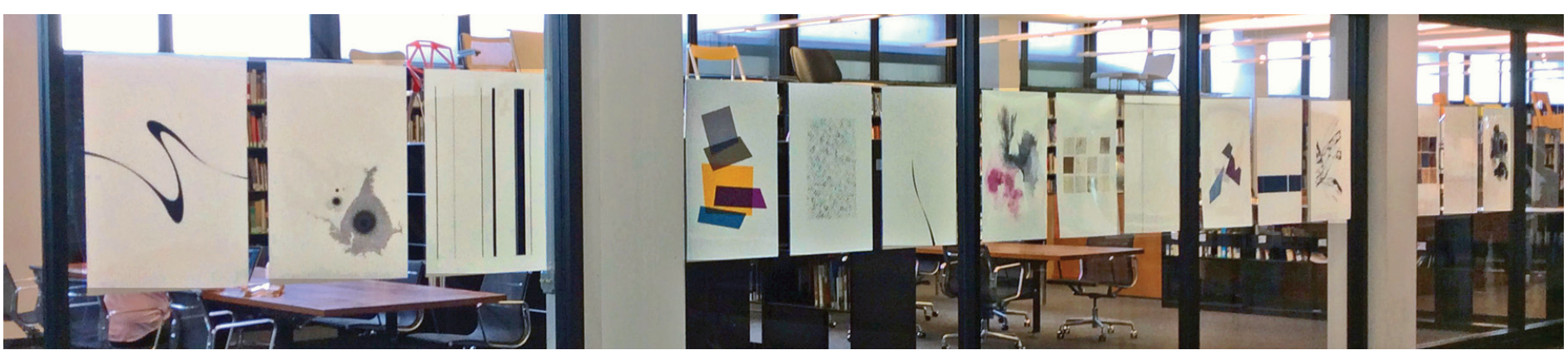

Figure 4. Visual Training exhibit, IIT 2015. (Image by author).

7 Hundreds of plates are contained in the collection ranging in age from 1955-current work. 
more picturesque quality. Specific values are taken into consideration with respect to overall pictorial quality and the development of aesthetic qualities such as depth and movement provide a sense of engagement - of entering into and participating in the image. The more picturesque examples can be viewed as more developed aesthetic expressions because they demonstrate advancement from seeing isolated elements and relations to an intuitive awareness of the dynamic whole.

Among these more picturesque examples, a myriad of variations are found. Some are heavier and some are lighter. Some exhibit more movement, some less. This difference in appearance may seem to contradict the notion of "objectivity" with respect to an aesthetic ideal because it opposes the conception of the aesthetic object as absolute or static. However, if the aesthetic object is not seen as absolute or static, it can be understood to have different modes of presentation without this difference being seen as a contradiction. In much the same way that a delicate wine is not necessarily better than a robust wine, and the quality of delicateness in a wine does not guarantee its excellence, the objective ideal of these exercises is in flux.

Other reasons accounting for visible differences in the outcomes can be attributed to specific instructors. Some variations have only been conducted by one instructor. For example, Peterhans taught the exercise with interlaced rhythms using two rows of spaces at Hochschule für Gestaltung in Ulm, and three rows at IIT. The trained eye will also notice that some instructors have tended toward finer line weight or more complex solutions, while others have tended toward simpler or more robust solutions. Peterhans was fascinated by the extremes and the sublime, for example - the nearly opposite and the almost symmetrical, whereas others were more inclined to the picturesque.

These differences in the course outcomes, therefore, do not necessarily justify the assumption that quality in art is altogether subjective and idiosyncratic, or further, that aesthetic judgment is unteachable. Rather than being seen as inconsistencies in an ideal, the differences in the Visual Training plates illustrate the pedagogical aspect of the exercises. In fact, the differences in the outcomes of the Visual Training course are equally as significant as what they have in common, as they tell the pedagogical story.

With each Visual Training exercise, students learn to understand possibility and ideal. To be in a position to guide students without hampering their development requires the ability to mentor. An instructor must be able to see how to improve a solution, and then strike a balance between demonstrating and asking the right questions in order for the student to arrive at real understanding and independence. The examples illustrate why an instructor's perspective on possibility and ideal needs to be both continuous and liquid, such that the potential is fluid but the aim is clear. The Visual Training program is clearly structured to address the fundamentals, but flexible enough for instructors to tailor the direction of each exercise to match the individual and collective needs of the students.

\section{THEORY OF VISUAL TRAINING Course Structure}

The background of the Visual Training course structure is embedded in its historical lineage. Since the founder of the course was a member of the Bauhaus faculty, it may be no surprise that precedents for each of the Visual Training exercises are found at the Bauhaus in the Vorkurs, or preliminary course. However, no course at the Bauhaus appears to have used the same combination of exercises or manner of instruction used at IIT. What Visual Training and the Vorkurs have in common is the foundational principle of the course. Peterhans' writings indicate that the set of problems used in Visual Training was intended to touch on the most basic elements of form, the axioms of aesthetics, as it were. ${ }^{8}$

Visual Training is a foundation seminar which partly precedes, partly runs in parallel, to the specialized work of the painter, architect, artisan; it is conscious education for seeing and forming, for aesthetic experience in the world of proportion, shape, color, texture, space.

It consists of exercises that are abstract enough to demonstrate visual qualities (texture, space) isolated from each other, detached from the manifold relationships in which they occur in the visual arts and architecture; and which are outlined definitively enough by theme and variation to clear conditions which simultaneously bind and free them from these conditions; and rich enough to exist in their own right, by intensifying visual qualities to the highest pitch, so that they appear in pure, crystalline form, pushing to achieve the maturity and fullness of free harmony.

These exercises include the most general sources of the formal values of the fine arts and architecture, and the ideas and the world of concepts that are essential for the analysis and critique of artwork.

By its nature, this seminar can also serve specialized work. It stimulates, permeates and controls them, as exercises are repeated from time to time when their need is felt. Enter distance and depth return. (Peterhans 1953)

The significance of axioms is that they function both as basic foundations of applied knowledge as well as elements of a conceptual framework, or theory (Hilbert and Fang 1970). ${ }^{9}$ When Peterhans states that Visual Training is "conscious education for seeing and forming", he is referring to the critical function of a foundation course with respect to making that connection between applied and abstract knowledge. Basic formal concepts like space and material are not only fundamental to art and architecture, but also to language and cognition. If we consider the structure of the Visual Training course in terms of basic concepts like

\footnotetext{
${ }^{8}$ See Peterhans' Fragment on Aesthetics for more on his axiomatic approach to aesthetics (Peterhans 1961).

${ }^{9}$ David Hilbert (1862-1943), best known for axiomatizing the field of geometry, exerted a profound influence on Peterhans as one of his teachers at University of Göttingen in the 1920s.
} 
space and material as well as technique, we begin to see the realm of aesthetics laid out by the exercises in connection with its theoretical purpose.

The exercises investigate the meaning of space by exploring the primary ways we define it. These ways include elements like line and plane, and relations such as orthogonality, continuity, and discontinuity, as well as constancy and change. The exercises dealing with sheets of paper question spatial definition by finite means, like subdivision, and by infinite means, like pictorial depth and view. The ink exercises also explore notions of continuity and discreteness, albeit in a "painterly" fashion, dealing with particles and more nebulous boundaries, or fields. Most of the exercises investigate two-dimensional space and the nature of pictorial or graphic space, but the three-dimensional exercise investigates the development of a three-dimensional aesthetic unity arising from the interaction between matter and space.

Each of the definitions of space offers a distinct means of understanding, interpreting and communicating about our experience of being in the world. The Visual Training program provides a platform for investigating these foundational constructs. This knowledge of space can be directly applied in the development of floorplans and elevations where a finite amount of space is distributed and shaped. It can also be applied in building massing and site planning which occurs in a more nebulous space. Deepening our understanding of these spatial paradigms, including their limits, potential and dynamics, enhances the visual framework from which conceptualization and imagination is forged.

Considered in terms of material or physical states, we see that the paper and ink exercises deal with solids and liquids. Handling these materials develops a sense of static and fluid behavior. If the primary states of matter are solid, liquid and gas, the exercises dealing with space objects of different shapes related in such ways as to suggest freedom of movement or free suspension in pictorial space - can be conceived of as formally corresponding with a gas or continuous ether.

The material concreteness of the exercises gives the lessons a tangible aspect, visible proof which is readily accessible to observation. The combination of exercises works together on an elementary level to develop the ability to understand and manage both definite and indefinite aspects of form. The exercises working with paper provide experience with clear and distinct boundaries while the ink exercises develop a sense of fuzzier boundaries. Observation of ink behavior develops an understanding of the direct and indirect influence we can have on fluid expression. Exercises dealing with assemblies of various solid materials foster an appreciation of nuances in character including texture, color and finish and develop a sense of grouping and arrangement. These experiences develop sensitivity to both the overt and the invisible forces underlying material form.

In architecture, applications for knowledge about material quality range from coordinating the colors and textures of materials and finishes to dealing with impact of wind forces, arranging groups of buildings or orienting and massing a particular building on a site. Training students to work with paper, fluid media, and a variety of other materials develops practical skills including measuring, cutting, adhering, brushing, stamping, and assembling, among others. These technical skills are directly applicable to creating drawings and renderings, physical models and other presentation materials. They also develop sensitivities to material characteristics, types and finishes, and to craftsmanship. In the practice of architecture, these skills were considered essential when the course originated. However some have questioned the practicality of the course since we now work on computers in architectural practice rather than with our hands.

The exercises were originally conceived of as being foundational in both practical and ideal (or conceptual) terms. However, today Visual Training is an elective course, which suggests that it is now perceived as a luxury or enhancement. ${ }^{10}$ The reason that the exercises were also considered fundamental in ideal terms has to do with the relationship between technology and vision. Revisiting this relationship raises important questions about the aims and means of architectural pedagogy.

The idea that technology shapes our view of the world is not new. A traditional fine arts program was based on a triadic relationship of technologies: painting, sculpture and architecture. These disciplines mapped out a formal realm in terms we might now think of as 2D, 3D object, and 3D environment. In the early 20th century, Heinrich Wölfflin observed a difference between the absolute or linear expression of drafting and the fluid, multi-layered expression of painting (Wölfflin 1915). He coined terms for these archetypes like "malerische" or painterly, using differences implied by technology to explain distinctions in what seemed to typify ways of seeing through the eye of the draftsman and the eye of the painter. The inclusion of the ink exercises in the Visual Training program likely stems from the desire to combat a perceived predisposition toward an absolute or linear perspective of the draftsman and the desire to develop a more well-rounded eye.

These days, we are reflecting on the impact of computer technology on vision. Peter Weibel, of the Karlsruhe Center for Art and Media, writes:

The triumph of the visual in the twentieth century is the triumph of a techno-vision. [...]The Latin word "video", meaning "I see", referred to the activity of a subject. Today it is the name of a machine system of vision. This turn shows clearly that we have entered a new era of vision, the technical vision, the machinebased vision. Machines generate, transmit, receive and interpret images. Machines observe for us, see for us. The eye triumphs only with the help of machines. This mechanical perception has changed both the world and the human perception of the world. (Weibel 1998)

Weibel, like Wölfflin, describes a vision conceived of as being tied to technology. In this case, a vision which is entrusted, even subordinate, to computers.

\footnotetext{
${ }^{10}$ The IIT course bulletins show that Visual Training was a required course which
} was switched to an elective ca. 1996. 
Many architecture students today have more experience working with computers than with their hands and physical materials. This is certainly an advantage when enrolling in educational programs which aim to advance computer skills. However, if one of the original aims of Visual Training was to mitigate the linear and absolute ways of thinking implied by drafting through exercises dealing with painterly techniques, then perhaps we ought to consider whether the format of the Visual Training exercises might help foster more rounded thinking today and mitigate some of the undesirable effects of working predominantly with computers. The concreteness of the existing exercises demands ways of thinking which counteract what Weibel observed as today's tendency to subordinate our senses to machines. The Visual Training exercises challenge tendencies toward the overly computational thinking (e.g. needing an algorithm to begin solving a problem) and overly infinitenebulous thinking (e.g. thinking that nothing is permanent, so nothing is critical) which seem to typify thinking in the computer age. The eye opening and sharpening functions of the exercises provide means to understanding a problem when an algorithm is not available and ways of effectively dealing with uncertainty while working in new territory.

Looking at the spatial, material, and technical characteristics of the exercises illustrates the formal logic of the Visual Training course structure. Collectively, the formal outcomes quite literally (though nonverbally) illustrate the mental constructs derived from the most basic concepts which shape our ways of looking at the world (e.g. "linear thinking" and "layers of meaning"). These perspectives show how the discrete-absolute, continuous-liquid, and infinite-ethereal aspects of the exercises connect practical skills with ways in which we perceive, comprehend and manipulate thoughts and ideas, all of which contribute to the development of good judgment.

\section{Pedagogical Theory}

The knowledge gained by doing the exercises has an immediate and obvious practical impact. Cutting long, thin, consistent lines and deftly fluid curves certainly sharpens visual acuity. This knowledge or ability can be applied from the drawing board to the construction site. But what is perhaps less obvious is the significance of this knowledge with respect to an educational ideal.

In philosophy, vision and insight have historically been viewed as related; either in opposition (e.g. Plato) or as complementary (e.g. Kant). The idea that the purpose of education is to "awaken" the mind to its own insight traces back through Kant's epistemology to Plato's concept of anamnesis or "recollection". The idea of awakening the mind to insight is the origin of what we call "critical thinking" today, and the underpinning of the "Socratic Method" of questioning often used in teaching.

The process by which insight is achieved remains a central question in education. In the interest of understanding the conditions for and nature of insight, the term "aesthetic" was coined in the context of German epistemology as a key form of judgment, distinct from reason and logic, and based on sensory knowledge. But whereas Plato contended that vision (i.e. observation) was not a reliable source of knowledge, according to Kant's philosophy, insight occurs as a product of transcending knowledge of observed particulars to universal knowledge. A combination of reason, logic and aesthetic judgment was thought to bear on true understanding, or insight. ${ }^{11}$ Both agreed, as do many educators today, that the path to insight does not follow a formula. Today's emphasis on critical thinking stems from the assumption that insight begins with a hunger or search for knowledge, and that rote learning leads to the phenomenon that Kant labeled a "dogmatic slumber" back in the 18th century (Kant and Ellington 2001).

Following Kant, vision was taken up as a major theme in educational reform throughout Europe and the U.S. in the 20th century. In childhood education, for example, Montessori's method promoted "first the education of the senses, then the education of the intellect" (Montessori 1964). In collegiate arts education, Wassily Kandinsky spoke of "teaching to see" (Kandinsky 1978), Josef Albers of "opening eyes" (Albers 1968), and Gyorgy Kepes of "educating the eye" (Kepes 1965). And at IIT, Peterhans introduced Visual Training.

Visual Training presumes that sensory knowledge can be a path to insight. Peterhans' Visual Training program is based on the theory that knowledge of formal possibilities and ideals informs aesthetic judgment and can be learned much like abstract mathematical concepts such as "number" are learned using concrete means; differences in levels of understanding notwithstanding, (Peterhans, n.d.). The exercises use simple concrete means not only to develop specific technical skills, but also to develop conceptual knowledge. Although concrete exercises provide case-specific knowledge based on direct observation and experience, the abstract subjects of the exercises (e.g. lines versus parts of a building) enables them to serve as foundations for abstract knowledge that is widely and generally applicable. The exercises can be used to illustrate aesthetic concepts like balance and movement, or aesthetic values like fitful or languid. A student comes up with the answers themselves to demonstrate understanding, just as they would in math.

The pedagogical approach used in Visual Training, facilitated by questioning, comparative analysis and critique, is well established in many areas of study. Using this approach to develop aesthetic judgment, however, is controversial. To the proponents of "free expression" in architecture school, it can appear rather restrictive, dogmatic even. ${ }^{12}$ Paradoxically, Visual Training is not meant to stifle free expression, but to develop an understanding of formal problems and the potential for expression. The approach is unique in architectural education not because it facilitates critical thinking with visual examples, but because it takes a student's own work rather than only historical examples to facilitate critical analysis.

In architecture school today, analysis and design are often taught in separate courses, for example, history and design studio, and the two need not be aligned by a single interpretation of architecture. Such

\footnotetext{
${ }^{11}$ See Kant's Critique of Pure Reason , Critique of Practical Reason, and Critique of Judgment.

${ }^{12}$ For Dennis Domer's discussion of this criticism, see "Walter Peterhans and Visual Training at Illinois Institute of Technology," (Domer 1992).
} 
misalignment is sustained in contemporary culture by compartmentalized views of the nature of aesthetic judgment (e.g. beauty) and the nature of scientific judgment (e.g. truth). According to the Stanford Encyclopedia of Philosophy:

The nature of beauty is one of the most enduring and controversial themes in Western philosophy, and is - with the nature of art-one of the two fundamental issues in philosophical aesthetics. Beauty has traditionally been counted among the ultimate values, with goodness, truth, and justice. It is a primary theme among ancient Greek, Hellenistic, and medieval philosophers, and was central to 18th and 19th-century thought []. By the beginning of the twentieth century, beauty was in decline as a subject of philosophical inquiry, and also as a primary goal of the arts. However, the last decade has seen a revival of interest in the subject... (Sartwell 2014).

Visual Training is set apart from other visual coursework found in architectural education because it does aim to reconcile aesthetic and scientific perspectives, rather than promote one or the other.

A favorite aphorism of Mies was "Beauty is the splendor of truth", a catholic saying which has been attributed to St. Augustine after Plato. The proclamation is based on the perception of a sublime interrelation between beauty and truth. It is along similar lines that Visual Training presumes that all works of art hold an inherent truth which speaks for itself and can be judged. Works are judged not only in the here and now, but also by a formal language and ideal which transcend time and place. This implicit and enduring truth - what Peterhans called the "original unity of construction and form"13 - is what Visual Training aims to reveal, and the pursuit of this truth is what makes some of the final plates so beautiful. In architecture, this understanding of expression informs not only the reading of historical form but also the reading of current design.

\section{RESEARCH IMPLICATIONS}

This paper outlines the program of exercises used at IIT for Visual Training and makes connections with the broader educational theory which underpins the teaching practice. In doing so, it reveals the significance of the course and teaching methods. But while justifications for the Visual Training have been made in both practical and theoretical terms, the question remains as to why Visual Training remains outside of current trends in architectural education.

One of the barriers facing Visual Training is the paradigm we use to define our programs. Whereas Peterhans saw Visual Training as aligned with Delight, or Venustas within Vitruvius' architectural paradigm (i.e. Commodity, Firmness, Delight) (Peterhans 1955), today our curricula are largely shaped by accreditation criteria. The National Architectural Accrediting Board (NAAB) assesses knowledge in four different educational realms: A. Critical Thinking and Representation, B. Building Practices, Technical Skills, and Knowledge, C. Integrated Architectural Solutions and D. Professional Practice (National Architectural Accrediting

\footnotetext{
${ }^{13}$ In his day, Peterhans dealt with this embedded and enduring truth in his
} History and Analysis of Art courses (see IIT Bulletins).
Board, Inc. 2014). Notably, there is no category addressing vision or the training of the eye. The best category for Visual Training seems to be Realm A. Student learning "aspirations" in Realm A include "Being broadly educated," "Valuing lifelong inquisitiveness," "Communicating graphically in a range of media," "Assessing evidence," "Comprehending people, place, and context," and "Recognizing the disparate needs of client, community, and society".

Although a niche for visual communication is carved out in the context of graphical communication, none of the learning aspirations directly addresses visual acuity or aesthetic judgment. Interestingly though, the reason that representation and critical thinking are recognized as one domain in Realm A does have to do with vision. As mentioned before, vision and insight have historically been viewed in relation to each other in philosophy. Representation and critical thinking correspond with Plato's Form and Idea. However, the Platonist claim is that true knowledge is of the intellect, which is eternal, and that knowledge by the senses (e.g. vision) yields only situationally useful, and therefore faulty, belief (Plato Phaedo 103E, 65d, Republic 514a). The extreme Platonist view is that noesis, or insight, can only be achieved without depending on faulty sensory knowledge. This line of thought eventually led to Plato's own rejection of art as a worthless pursuit and is the reason that in some circles science is considered a more respectable pursuit than art. Kant held a more moderate view; that insight occurs as a product of transcending knowledge of observed particulars to universal knowledge. The latter view was not only the grounds for the 20th century educational reform movements mentioned earlier, (e.g. Montessori Method and the Bauhaus pedagogy), it is the enduring theoretical justification for education in the arts.

Assuming that observation can lead to insight, Visual Training's concrete problems exercising visual perception make perfect sense toward developing aesthetic judgment in an abstract or general sense. Unfortunately, Plato's dilemma still confounds our schooling. Reinforced by 20th century right-left brain theory, we have fortified a barrier between the teaching of arts and sciences. Separate paths have been paved for pursuing beauty and truth in education, and the two seldom meet. Even in philosophy, we find separate tracks for philosophy of science and philosophy of art. Furthermore, the treatment of the arts as disposable subjects in our schools and sensory-related intelligence as extra-curricular indicates a bias in our current educational model. ${ }^{14}$ In our schools, we are systematically undermining the power of art to communicate higher understanding by separating it from the sciences. Some have begun to question whether this bias is to blame for our contemporary woes. As visual theorist and arts educator, Rick Williams, wrote:

Perhaps the void that many individuals experience and express, as well as unsolved answers to many personal and cultural problems, resides, to a significant degree, in the empty spaces of the untapped potential that balancing the use of our visual/ synthesistic and rational/analytical intelligences and cognitive

\footnotetext{
${ }^{14}$ Also see "Cognitive Theory" (Williams 2011) for discussion of the left-brain bias
} in our educational culture. 
abilities would realize. I suggest that ... rational biases contribute to visual and intuitive illiteracy and thus to the effective proliferation of persuasive ... media-generated communication. (Williams 2011)

Tracing the role of vision in our culture, from today's multi-faceted visual theory back to the philosophical debate on vision and insight, we begin to appreciate the complex of issues surrounding vision in education and how these issues are shaping our current programs. Much of our educational programming is based on a dualistic image of truth and beauty (with a rational bias) rather than an integrative one. However, current research seems to suggest that an alternative model is needed.

This paper reveals that in architectural education, Visual Training provides an example of pedagogy aimed at developing an often overlooked aspect of knowledge at the intersection of science and art which is central to judgment. Tangible lessons capture the essence of our most fundamental concepts as a means to our highest educational aspirations. Applications for the knowledge, skills and understanding gained by doing the exercises extend from production skills, through general creative and analytical thinking, to independent aesthetic judgment as grounds for architectural critique. Although Visual Training overlaps with topics in Visual Communication and Visual Design, it can be distinguished by its focus on developing an understanding of beauty which makes a connection between the observed particulars and general or abstract knowledge toward insight. The existing program, with its field-tested pedagogy and documented results, undoubtedly harbors further evidence and potential waiting to be tapped.

\section{REFERENCES}

Albers, Josef. 1968. Interview with Josef Albers. Interview by Sevim Fesci. Tape-recorded interview. Archives of American Art. Smithsonian Institution.

Coppola, Francis Ford. 2016. "Coppola and The Art of Coffee." Wine Spectator, September 30.

Dearstyne, Howard. 1944. "Basic Teaching of Architecture." Liturgical Arts 12 (May): 56-60.

Domer, Dennis. 1992. "Walter Peterhans and Visual Training at Illinois Institute of Technology." The Structurist, on Structure and Color in Space, no. 31/32: 44-51.

Fry, Roger. 2011 (Rev. ed). Vision and Design. Mineola, N.Y: Dover Publications.

Hilbert, David. 1970 (in English). "Axiomatic Thinking." Translated by J. Fang and reprinted in Philosophia Mathematica VII (1-2): 1-12.

Kandinsky, Nina. 1978. Kandinsky et moi. Paris: Flammarion.

Kant, Immanuel, and James Wesley Ellington. 2001. Prolegomena to Any Future Metaphysics (Second Edition): And the Letter to Marcus Herz, February 1772. Hackett Publishing.

Kepes, Gyorgy. 1965. Education of Vision. New York : G. Braziller. PMCid:PMC495856
Mies van der Rohe, Ludwig. 1965. "Peterhans' Visual Training Course at the Architectural Department of IIT." Chicago.

Montessori, Maria. 1964 (Rev. ed.). The Montessori Method. New York: Schocken Books.

National Architectural Accrediting Board, Inc. 2014. "2014 Conditions for Accreditation."

Peterhans, Walter. 1953. "Peterhans Introduction." TS. UIm, Germany. Walter Peterhans Collection. HfG-Archiv Ulm.

Peterhans, Walter. 1955. "Visual Training." Dimension: University of Michigan Press. PMid:13297335

Peterhans, Walter. 1958. "Outline for Introduction of Visual Training Problems." TS. Walter Peterhans Collection. IIT Archives.

Peterhans, Walter. 1961. "Fragment on Aesthetics." Ratio, Basil Blackwell, Vol. III.

Peterhans, Walter. n.d. "On the Nature of Aesthetic Evaluation: General Summary of Problems Proposed for Investigation." TS. Nachlaß Peterhans: Manuskripte: :Asthetik \#1. Museum Folkwang Essen.

Plato. 1974 (Rev. ed.). Plato's Republic. Indianapolis : Hackett.

Sartwell, Crispin. 2014. "Beauty." In The Stanford Encyclopedia of Philosophy, edited by Edward N. Zalta, Spring 2014. http://plato. stanford.edu/archives/spr2014/entries/beauty.

Weibel, Peter. 1998. "The Unreasonable Effectiveness of the Methodological Convergence of Art and Science." In Art @ Science, edited by Christa Sommerer and Laurent Mignonneau, 167-80. Austria: Springer-Verlag/Wien. PMCid:PMC2212147

Utsunomiya, San. 1977. "Visual Training." unpublished manuscript, 2 pp. with 17 illustrations.

Vitruvius, Pollio. 2005 (in English). Ten Books on Architecture. Translated by Morris Hicky Morgan. Stilwell, KS: Digireads.com Publishing.

"Welcome to Visual Studies | Visual Studies." 2016. Accessed February 9. https://www.sas.upenn.edu/visual-studies.

Williams, Rick. 2011. "Cognitive Theory." In Handbook of Visual Communication: Theory, Methods, and Media, edited by Ken Smith. New York: Routledge.

Wölfflin, Heinrich. 1950 (Rev. ed.). Principles of Art History: The Problem of the Development of Style in Later Art. Translated by Marie Donald Mackie Hottinger. New York: Dover. 